\title{
Automatically Connecting Hardware Blocks via Light-Weight Matching Techniques
}

\author{
Jan Malburg* \\ Niklas Krafczyk* \\ *Institute of Computer Science \\ University of Bremen \\ 28359 Bremen, Germany \\ \{malburg,thurisaz\}@informatik.uni-bremen.de
}

Görschwin Fey*†

\author{
${ }^{\dagger}$ Institute of Space Systems \\ German Aerospace Center \\ 28359 Bremen, Germany \\ Goerschwin.Fey@dlr.de
}

\begin{abstract}
In modern chip design, many different blocks are assembled in a single chip. Normally, these blocks have been written by different developers or even licensed from other companies. Correctly connecting all blocks is a tedious task. State of the art tools for automatically generating the connections either require identical port-names or additional user input describing the intended connections.

In this paper we present an automatic approach for connecting different blocks. In contrast to previous approaches, we neither need exact name matching of the port-names nor additional user input. An evaluation showed the advantages of our approach. For seven of eight designs our approach generated better connections than a previous approach, including a design which has been optimized for being used with the previous approach.
\end{abstract}

A second goal of this paper is to understand the limitations of the presented light-weight matching techniques.

\section{INTRODUCTION}

Modern chip designs are composed out of several different blocks. Blocks are typically described in a Hardware Description Language (HDL). Often these blocks are from different developers or even third party blocks licensed from other companies and have to be assembled into a single chip. Writing the corresponding connections is a tedious task for a developer, as he needs to connect several hundreds or even thousands of different ports. Hence, automation is desirable.

We consider a technique as automatic if it only requires the blocks and the number of instances of each block. In this sense tools are not considered automatic if they require further user input like descriptions of the intended connections as, for example, the tool MKTREE [1]. An example for an automatic tool based on this definition is the openly available Emacs Verilog-Mode [2], which allows automatic generation of the connections. Additionally, a developer can add hints to support the generation.

In this paper we propose a technique which automatically generates the connections between a set of blocks. Our technique uses heuristics to compute likely connections between the different blocks using the similarities of the port-names, the bit-width of the ports and the datadirection. In contrast to similar techniques, our technique does not require $100 \%$ matching of the port-names to find the connections. This is an advantage, as often there is no $100 \%$ match of the names, either because the blocks are from different companies with different naming conventions or the naming convention includes pre- and suffixes for the data-direction and -width. A secondary research goal of this work is to evaluate the limitations of light-weight approaches for automatically connecting modules. Therefore, no computation intensive functional techniques are used.

We evaluated our approach on eight different designs of different size, authors, functionality, and origin. As comparison we use Emacs Verilog-Mode. With respect to a quality metric, which approximates the amount of work such a tool saves a developer, our approach generates equal or better connections than Emacs Verilog-Mode for all of the eight designs considered. This even includes a design which has been optimized for being used with Emacs Verilog-Mode. For one

This work was supported in part by the German Research Foundation (DFG, grant no. FE 797/6-1) design, for which Emacs Verilog-Mode was not able to create any connections, our approach generates a perfect set of connections.

Finally, we present cases which cause poor results for the approach and possible future improvements.

The remainder of this paper is organized as follows: Section II describes related work. Our approach is described in Section III including the different heuristic and connection strategies we are using. In Section IV we present the used quality metric and the evaluation of our approach. In Section V the limitation of the evaluated lightweight techniques and future improvements are discussed. Section VI concludes the paper.

\section{RELATED WORK}

In this section we discuss existing tools for generating connections between ports of different modules. However those techniques either require exact name matching of the port names or additional user input.

With SystemVerilog 1800-2005 [3] the implicit port instantiation operators . * and . name have been introduced. The operators allow shortening the instantiation list of modules. However, they require a complete match of the signal names with the port-names and that the signals are already defined in the module. In contrast, our approach neither requires that signals are already defined nor that there is a $100 \%$ matching between the names. The implicit port instantiation operators and our approach have in common that they expect correct data-width and data-direction.

The Emacs plugin Verilog-Mode [2] adds several options for automatic instantiation to the text editor Emacs. The option AUTOINST allows instantiation based on direct name matching similar to the .* operator for SystemVerilog. Further, a user can use regular expressions in order to lift the requirement of exact name matching. Additionally, Verilog-Mode provides AUTOWIRE and AUTOREG which automatically declares wires and registers based on the module's input and output definitions. Still this approach either requires exact name matching or complex user input, in form of the corresponding regular expressions, to automatically create the port connections. Similarly, Emacs plugins for VHDL exist as well [4].

MKTREE [1] is a tool to create connections between several Verilog modules. For this MKTREE utilizes a special description language to reduce the coding effort for a developer. Therefore, MKTREE is not an automatic tool in our sense and has the disadvantage that the developer has to learn the description language used.

ShapeUp [5] is a tool which uses a connection description written in Click, a language originally designed to describe network systems for generating the connection between modules. Further, ShapeUp utilizes module-interface descriptions based on the IP-XACT standard [6] in order to ensure correct wiring between the different modules. To some extend ShapeUp is even able to automatically generate converter blocks in cases where the interfaces do not completely match, for example in presence of different bit-widths. However, both the Click description of the intended connection and the IP-XACT description of the modules need user interaction. Thus ShapeUp is not considered an automatic tool in our sense. 


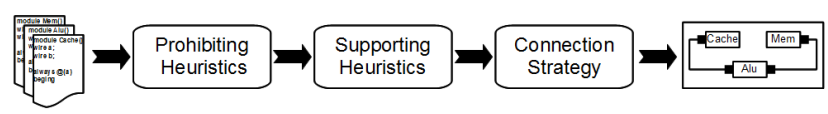

Figure 1. The basic flow of our approach

In [7] Avnit et. al. present an approach to automatically generate protocol converters for the communication protocols of two blocks. Their approach needs a description of source protocol and target protocol in form of a finite state machine and a mapping of dataand control-ports. Based on this they compute a hardware block implementing a finite state machine, which translates one protocol into the other. Their approach differs from ours as we assume matching protocols where they explicitly assume nonmatching protocols. Further, their approach needs user input in form of the finite state machines and the port mapping and uses computational costly formal analysis.

\section{TEChNIQUE}

In this section we describe our technique and the heuristics we are using. The implementation of our technique is based on the parser of IcarusVerilog [8]. Consequently, our implementation only supports Verilog. However, the basic idea should work for other HDLs, like VHDL or SystemC as well.

The basic flow of our approach is shown in Figure 1. As input a list of Verilog modules, the source code of each module, and, optionally, the amount how often each module should be instantiated is used. First, a set of prohibiting heuristics is applied on the modules. The prohibiting heuristics mark connections as forbidden either because they are very unlikely or would result in nonsynthesizable code. Then supporting heuristics are used which compute the likelihood of a connection of being correct based on name comparison. The last step in the computation is the application of a connection strategy which uses the results of the heuristics. Some strategies additionally use a threshold or a safety approximation. The connection strategy returns the generated connections between the different modules.

\section{A. Prohibiting heuristics}

In this section we present the heuristics which mark connections between two ports as forbidden connections either because such a connection would result in invalid Verilog code or the connection is extreme unlikely.

- No output-to-output connections:

Such a connection is not allowed by Verilog.

- No Output-to-InOut connections:

A connection of an InOut port with an Output-port is valid in Verilog. But this type of connection is unlikely and might often result in multiple driver errors during synthesis.

- No connection between ports with different bit-width:

Like the previous case, this results in correct Verilog code but is rather unlikely.

- No connection between two ports of the same module-instance: This is also very unlikely as the information could be forwarded inside the module.

- No connection of a port with itself:

Such a connection cannot be expressed in Verilog.

\section{B. Supporting Heuristics}

Here we present several heuristics to compute a likelihood for two ports to be connected. These heuristics assign values to pairs of ports in the range from zero to one, where zero means unlikely and one means very likely.
1) Name matching $(\mathrm{Nm})$ : The first set of heuristics we present is based on the similarity of the port-names. For any sensible naming convention for port-names, those names should contain information about the data, which is expected to be send over that port. Consequently, if two ports should be connected, the same data is to be send over both ports. Over one of the ports as input and over the other port as output. Thus it can be assumed that the ports which should be connected have to some extend similar names.

We implement three different heuristics based on the stringsimilarity metrics Jaro-distance [9], Levenshtein-distance [10] and longest-common-substring.

- Jaro-distance $\left(\mathrm{Nm}_{J}\right)$ :

For computing the Jaro-distance, first the relation of matching characters gets computed, where characters are considered matching if they are identical and their position does not differ beyond a value defined by the length of the string. Then the amount of transpositions is counted. A transposition is a set of two matching characters, which are in different order in the two strings. After that, the distance is computed as the average of the relation of the matching characters to the length of the strings and the relation of matching non-transposed characters to matching characters.

- Levenshtein-distance $\left(N m_{L e v}\right)$ :

The Levenshtein-distance, also known as edit-distance, is defined as the minimal number of edit operations which have to be applied in order to change one string into the other. An editoperation is defined as either, deleting a character, adding a character, or the replacement of a character. The largest possible Levenshtein-distance of two strings is the length of the longer of those two strings.

- Longest-common-substring $\left(N m_{L C S}\right)$ :

The longest-common-substring is the length of the longest substring which both strings have in common. This value then is normalized based on the average size of both strings. The idea in using the longest-common-substring is that in many namingconventions characteristics of the port, like direction and bitwidth, are included in the name in form of a pre- or suffix.

For our heuristic computation the result of the distance computation is normalized to the range between zero and one, where zero means no similarities between the strings and one means identical strings. The normalized value is the resulting likelihood.

2) Extended name matching $(E N m)$ : Like the normal name matching, but additionally, the port name is also compared to the module name of the ports to which it should be connected, the submodules, if any, which use the port and the port names for those submodules. The maximum score of all these computations is used. As for name matching extended name matching can be combined with the different string metrics resulting in the three heuristics $E N m_{J}, E N m_{L e v}$ and ENm $m_{L C S}$.

3) Event checking (Ev): In synchronous designs, there exists always a clock signal and most likely a reset signal. If the event checking heuristic is used, a top level signal clock is assumed to exist as well as a signal reset. The sensitivity lists of the modules and submodules are checked, and it is assumed that reset and clock signal are signals which appear in the sensitivity lists of the modules. For those signals which appear in the sensitivity list, their names get compared to the names clock and reset, as normally the clock signal is named clock or clk and the reset signal reset or rst. The result of this comparison is used as the assigned likelihood. Because, this heuristic only compares port-names with clock and reset, the heuristic only assigns likelihoods to such connections.

\section{Score and Threshold}

Over all heuristics a score is computed for the connection between two ports. For this the likelihoods computed by the different heuristics get summed up. Also the prohibiting heuristics assign likelihoods 
to the possible connections, hereby the following rules apply: The heuristic which checks for port direction assigns 0.5 to connections between input and InOut ports and to all other legal connection the likelihood 1.0. Forbidden connections get the likelihood 0.0 assigned. Correspondingly, the heuristic which checks for port width assign a likelihood of 1.0 to connections with matching port widths and 0.0 otherwise.

A threshold on the computed score has been defined in order to decide if a connection is likely or not. As the likelihood of all used heuristics get summed up, the maximal score value depends on the number of used heuristics. Therefore, the value of the threshold is defined as a fraction of the maximum score. Preliminary experiments showed that choosing a threshold equal to two third of the maximal reachable score is effective; thus this is the value we are using for the experiments in this paper.

\section{Safety Approximation}

For a port, there are often several ports to which it can connect. However, input ports are only allowed to connect to a single other port. All those possible ports present alternatives to which the port can be connected. Potentially, some of those connections might not have the highest score, but do not have any other likely connection and the connection with the highest score may have many different other possible connections. In this section we present a safety approximation which also considers the score of alternative connections. The basic idea of the safety approximation is to compute, how much more likely a connection is compared to the other connections the associated port could be part of. Let $s(i, j)$ be the score for the ports $i$ and $j$. Further, let $s_{\max }$ be the highest possible score and $\mathcal{P}$ the set of all ports. Then we define the safety approximation $c(i, j)$ between the port $i$ and $j$ as:

$$
c(i, j)=b(i, j) * \frac{s(i, j)}{s_{a v g}(i, j)} * \frac{s(i, j)}{s_{\max }}
$$

With $b(i, j)$ is a factor based on the prohibiting heuristics:

$$
b(i, j)= \begin{cases}0 & \text { connection marked as forbidden } \\ 1 & \text { otherwise }\end{cases}
$$

and $s_{a v g}(i, j)$ is the average score which $i$ and $j$ reach:

$$
s_{\text {avg }}(i, j)=\frac{1}{2 *|\mathcal{P}|} * \sum_{k}^{\mathcal{P}}(s(i, k) * b(i, k)+s(j, k) * b(j, k))
$$

\section{E. Connection Strategies}

We implemented several different strategies for choosing the connections between the ports

1) Greedy approach using the score $\left(S_{h f}\right)$ :

Always the connection with the highest score is created first. This might make other connections invalid. Connections are created until no further connection with a score higher than or equal to the threshold is possible.

2) Greedy approach using the safety approximation $\left(S_{c m}\right)$ : Like $S_{h f}$ but creating the connection with the highest safety approximation first.

3) Preferring already connected modules using the score $\left(S_{e m, h f}\right)$ : Like $S_{h f}$, however when choosing the next connection to create, for connections between two modules which already are connected, the scores of these connections get multiplied by 1.1 . This is due of the fact, that often a package of logical information is sent over a combination of several ports.

4) Preferring already connected modules using the safety approximation $\left(S_{e m, c m}\right)$ : Like $S_{e m, h f}$, but using the safety approximation instead of the score.

\begin{tabular}{l|r|r|r|r} 
Name & module \# & port \# & $n_{\text {orig }}$ & Source \\
\hline SHA3 & 3 & 26 & 11 & {$[11]$} \\
Wishbone-specification & 3 & 38 & 20 & {$[12]$} \\
SD Mass Storage Controller & 4 & 60 & 10 & {$[13]$} \\
uart2spi & 4 & 61 & 31 & {$[14]$} \\
tiny-AES & 27 & 115 & 66 & {$[15]$} \\
MIPS789 & 19 & 140 & 83 & {$[16]$} \\
OpenRISC 1200 & 14 & 429 & 230 & {$[17]$} \\
OpenSPARC T1 core & 12 & 1230 & 626 & {$[18]$}
\end{tabular}

Table I

OVERVIEW OF THE DESIGNS USED FOR OUR EVALUATION

\section{Evaluation}

In this section we evaluate our approach on several designs. In order to reduce possible bias we use designs of different size, of different purpose, and from different authors. For the evaluation we removed the sub-module instantiations from the top-modules of the designs and then applied our approach to recreate the instantiations. We used Emacs Verilog-Mode as a baseline-comparison. We have chosen Emacs Verilog-Mode because it is a freely available tool, which uses similar inputs as our approach, however a developer may add hints, in form of regular expressions, to improve the result. A connection is considered correct if it is identical to a connection of the original top-module; otherwise the connection is considered incorrect. Thus, the resulting connection is optimal, if and only if it is semantically equivalent to the original top-module.

For comparing the results a quality measurement has been defined. Let $n_{\text {corr }}$ be the number of correctly generated connections, $n_{\text {all }}$ the number of all generated connections and $n_{\text {orig }}$ the number of expected correct connections. Our quality measurement $q$ is then defined as:

$$
q=\frac{\frac{n_{\text {corr }}-\left(n_{\text {all }}-n_{\text {corr } r}\right)-\left(n_{\text {orig }}-n_{\text {corr }}\right)}{n_{\text {orig }}}+1}{2}
$$

The intention of this measurement is to approximate the amount of work a developer can save by using a tool ${ }^{1}$ for creating the connections: $n_{\text {corr }}$ is the amount of correct connections, i.e., work a developer has saved; however, a developer has to remove the connections the tool has incorrectly created $\left(n_{\text {all }}-n_{\text {corr }}\right)$ and, finally, he has to add all missing connections $\left(n_{\text {orig }}-n_{\text {corr }}\right)$. The rest of the formula normalizes the value such that 0 means no work is saved, corresponding to the case of the design without any connections, and 1 means that all connections are correctly generated, i.e., all work is saved. The formula may return negative numbers; this is the case when the correction of the returned solution is harder than connecting the modules completely by hand.

Table I gives an overview of the designs used for this evaluation. SHA3 is a design for computing the SHA3-hashcode of the input data. The Wishbone-interface is an interface for register and memory access, used in many designs on the OpenCores.org-website. The interface allows a master and several slaves on a single bus. The specification includes the naming convention for the corresponding ports. The design Wishbone-specification only consists of submodules with the correspondingly named ports but without any actual logic. SD Mass Storage Controller is an SD-Card controller. The design uart2spi is a protocol translation block between UART and SPI which can additionally parse some simple commands directly. MIPS789 and OpenRISC 1200 are two different RISC processor designs. The OpenRISC 1200 supports floating point arithmetic and includes data- and instruction caches, a memory management unit, a timer, an interrupt controller, a debugging unit and two Wishboneinterfaces. OpenSPARC T1 core is a core of the OpenSPARC T1 processor. The OpenSPARC seems to be developed using the Emacs

\footnotetext{
${ }^{1}$ Approximation because it assumes that the work a developer needs to remove a wrong connection and to create a correct connection is identically. Under this assumption the value is a exact value.
} 


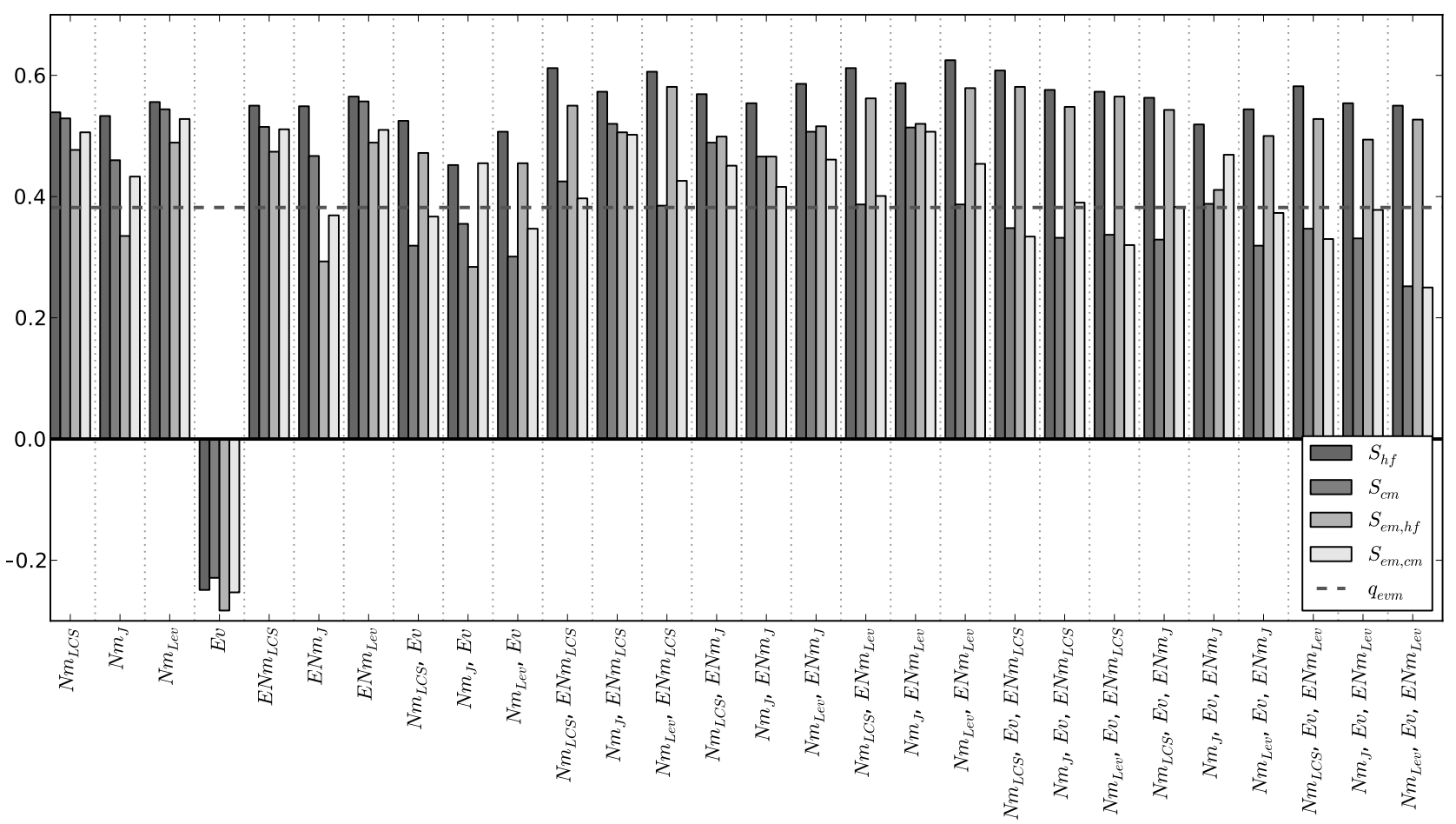

Figure 2. Average $q$-value for different heuristics and connection strategies

\begin{tabular}{l|r|r|r|r|r} 
Name & Parsing & $S_{h f}$ & $S_{c m}$ & $S_{e m, h f}$ & $S_{e m, c m}$ \\
\hline SHA3 & 0,05 & 0,01 & 0,01 & $<0.01$ & 0,01 \\
Wishbone-specification & $<0.01$ & 0,01 & 0,01 & 0,02 & 0,01 \\
SD Mas Storage Controller & 0,01 & 0,08 & 0,08 & 0,08 & 0,08 \\
uart2spi & 0,02 & 0,09 & 0,09 & 0,09 & 0,09 \\
tiny-AES & 1,18 & 0,30 & 0,30 & 0,30 & 0,31 \\
MIPS789 & 0,02 & 0,51 & 0,51 & 0,55 & 0,52 \\
OpenRISC 1200 & 0,10 & 4,76 & 5,27 & 4,95 & 5,53 \\
OpenSPARC T1 core & 19,61 & 95,80 & 79,48 & 98,28 & 79,93 \\
& Table II
\end{tabular}

PROCESSOR TIME FOR USING OUR APPROACH IN SECONDS, FOR THE DIFFERENT DESIGNS

Verilog-Mode as it includes the corresponding hints and the original module instantiation is in the form Emacs Verilog-Mode creates.

We have measured the runtime of our approach for the different designs. The measurement was conducted on an Intel Core2 Duo $2 \mathrm{GHz}$, running Archlinux 3.9.3-1 and all heuristics have been used. Table II shows the processor time our technique required. The column parsing gives the time required by the Icarus Verilog parser to generate the abstract syntax trees for the design with all its sub-modules. The technique has been applied as presented in this paper, without special optimization. As our approach requires that each port-name is compared to each other port-name the runtime of our approach is quadratic in the number of ports.

In the following paragraphs we present the results for our approach. Due to space limitations, we only present the average results over all designs (Figure 2) as well as the detailed results for the designs OpenSPARC T1 core (Figure 3) and tiny-AES (Figure 4). The OpenSPARC T1 core has been chosen because it includes hints for the Emacs Verilog-Mode and therefore is considered optimal for Emacs Verilog-Mode. The tiny-AES design was chosen because it shows results different from the average case.

Figure 2 shows the average $q$-values over all evaluated designs for different combinations of heuristics and connection strategies. The different combinations of the heuristics which are used are shown at the bottom. The abbreviations introduced in Section III-B are used. In all cases the preventing heuristics are used as well. The different connection strategies are differentiated by the bar-colors. The result for Emacs Verilog-Mode $\left(q_{\text {evm }}\right)$ is shown in the figure as dotted line. The best result with a $q$-value of 0.625 has been achieved by combining $N m_{L e v}$ and $E N m_{L e v}$ and using $S_{h f}$ as connection strategy. Further, $S_{h f}$ achieves the best average result for every combination of heuristics expect when used with the event checking heuristic only. Additionally, we see that using the event checking heuristic decreases the quality of the result on average.

As already mentioned the OpenSPARC design is especially interesting for a comparison between our approach and Emacs VerilogMode because the design is optimized for the usage with Emacs Verilog-Mode. This includes a corresponding naming convention and hints for the connection generation process. The results are shown in Figure 3. First, we notice that again $S_{h f}$ achieves the best results and is able to achieve larger $q$-values than Emacs Verilog-Mode for most combinations of the heuristics. This design nicely shows that coding conventions which help other automatic tools, or are even required for the tools to be used, help our approach as well. But our approach does not require a developer to add supplementary information for the tool, like the regular expression for the Verilog-Mode or the connection rules for MKTREE. Even in cases where regular expressions are used to define the correspondence of signals, like for Emacs Verilog-Mode, the port-names should still have a strong similarity, which is sufficient for our approach.

If we have a closer look at the connection strategies, we can see that the qualities of $S_{\mathrm{cm}}$ and $S_{\mathrm{em}, \mathrm{cm}}$ are heavily fluctuating. Changing a single heuristic can change the resulting $q$-value by 0.5 or more. For example, the combination of name matching with the Jaro stringmetric and extended name matching with Levenshtein achieves a $q$ value above 0.8 . However, by changing the string metric for name matching, the $q$-value drops below 0.1. Similar effects appear for the designs MIPS789, OpenRISC 1200 and to some extent for the designs SD Mass Storage Controller and Wishbone-specification. This shows that $S_{c m}$ and $S_{e m, c m}$ are rather unreliable and hence, not very useful.

Considering the results for tiny-AES design in Figure 4, we see that the result deviates from the other designs with respect to two attributes. First, the connection strategies $S_{c m}$ and $S_{e m, c m}$ achieve the best results for all combinations of heuristics. Second, using $E v$ 


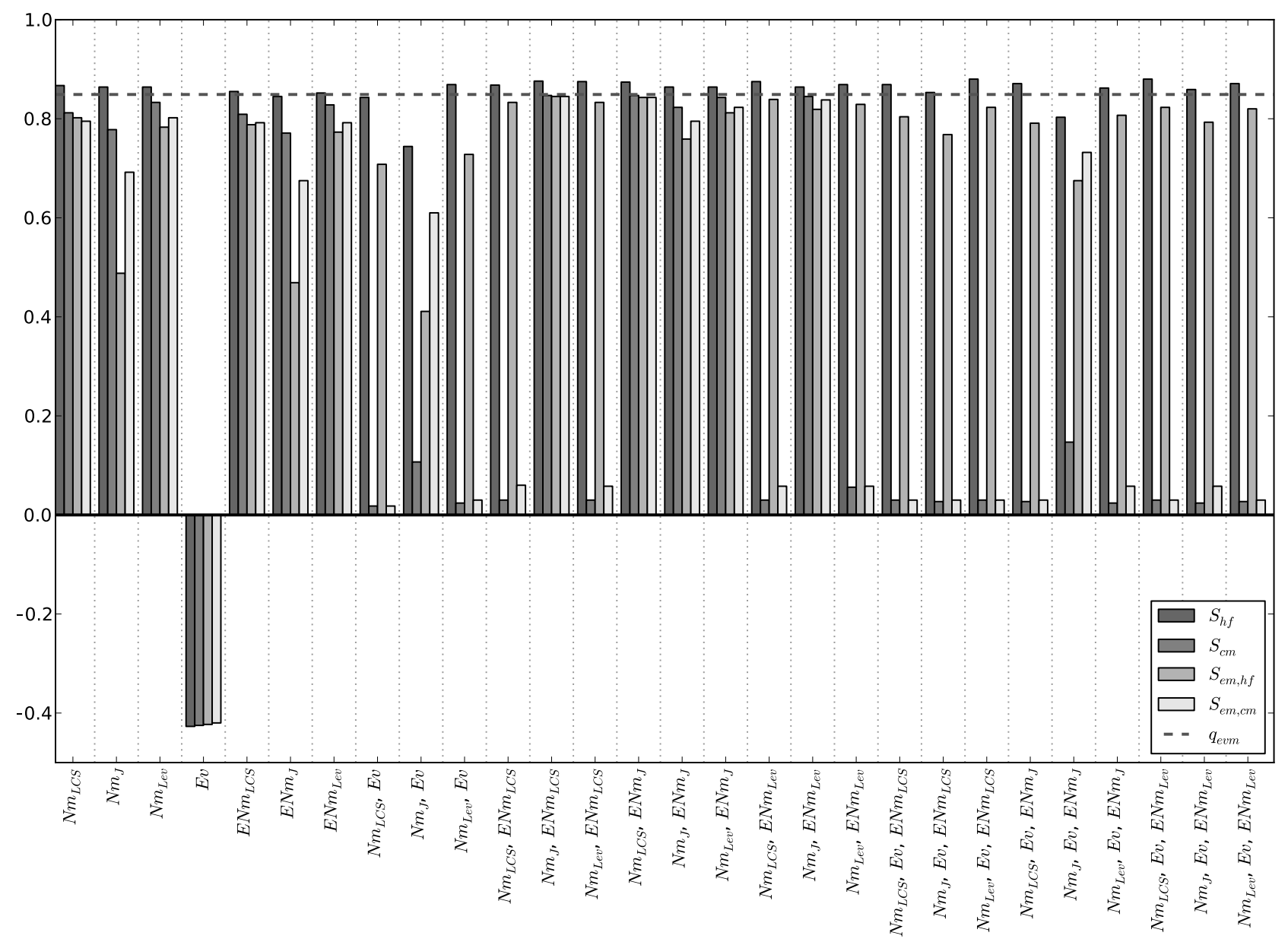

Figure 3. The $q$-value of the OpenSPARC T1-design for different heuristics and connection strategies

improves the quality of the results for the tiny-AES design in 26 of 48 of the cases and never reduces the quality of the result. In contrast, for the other designs using $E v$ only improves the result in very rare cases and in many cases even reduces the quality of the result. Interestingly, in all cases at least 27 correct connections has been created. This is the same number of connections Emacs Verilog-Mode creates. Also, the best results are those where no other connections have been created. In the cases where more than those 27 connections have been created, the quality of the additionally created connections is not better than randomly created connections.

Further, the Wishbone-specification is to mention. Our approach using $S_{h f}$ creates the correct wiring ( $q$-value equals 1 ) for any combination of heuristics which contain name matching or extended name matching. Further, $S_{e m, h f}$ is able to create an optimal result in most (15 out of 28) cases. The connection strategies $S_{c m}$ and $S_{e m, c m}$ only achieve $q$-values between 0.4 and 0.9 . Again those combinations achieve the lowest $q$-values which include the $E v$ heuristic.

Finally, we compare our approach with the results of Emacs Verilog-Mode. Table III gives the results when applying Emacs Verilog-Mode to the designs and values achieved by our approach. The column "highest" shows the highest achieved $q$-value over all combinations of heuristics and connection strategies. The column "best avg." shows the result for $N m_{L e v}$ combined with $E N m_{L e v}$ and the connection strategy $S_{h f}$, which was the combination achieving the highest average $q$-value. First, we notice that in case of the Wishbone-specification Emacs Verilog-Mode is not able to create any connection. This is due the fact that the naming convention used for the specification includes a suffix for the port direction. Hence, there is no exact name matching. In contrast our approach

\begin{tabular}{l|r|r|r|r|r} 
& \multicolumn{3}{|c}{ Emacs Verilog-Mode } & \multicolumn{2}{c}{ Our technique } \\
Name & $n_{\text {all }}$ & $n_{\text {corr }}$ & $q$ & best avg. & highest \\
\hline SHA3 & 12 & 9 & 0.682 & 0.818 & 0.909 \\
Wishbone-specification & 0 & 0 & 0.000 & 1.000 & 1.000 \\
SD Mass Storage Controller & 3 & 0 & -0.150 & 0.100 & 0.333 \\
uart2spi & 26 & 26 & 0.839 & 0.903 & 0.903 \\
tiny-AES & 27 & 27 & 0.409 & 0.318 & 0.409 \\
MIPS789 & 22 & 20 & 0.229 & 0.331 & 0.404 \\
OpenRISC 1200 & 46 & 46 & 0.200 & 0.663 & 0.754 \\
OpenSPARC T1 core & 542 & 535 & 0.849 & 0.869 & 0.880
\end{tabular}

Table III

THE RESUlts OF THE EMACS VERILOG-MOdE FOR THE DIFFERENT DESIGNS, COMPARED WITH THE PRESENTED TECHNIQUE, USING THE COMBINATION $\left(N m_{L e v}, E N m_{L e v}, S_{h f}\right.$ WHICH RESULT IN THE HIGHEST AVERAGE RESULT (BEST AVG.) AND THE HIGHEST OVERALL RESULT (HIGHEST)

generates a perfect result. Second, in case of the SD Mass Storage Controller Emacs Verilog-Mode only creates incorrect connections resulting in a negative $q$-value. Finally, in case of the OpenSPARC T1 core, although created to be used with the Emacs Verilog-Mode and corresponding hints are provided, incorrect connections are generated and again the technique presented in this paper achieved better result. Altogether we see that the presented approach is able to generate connections at least as good as Emacs Verilog-Mode and in many cases even better. Even as only the combination with the highest average $q$-value is considered, our technique achieves better results in seven of eight cases.

Additionally, the results for the Wishbone-interface, which represents one interface to combine blocks from different sources, suggest that our approach is effective for combining several blocks from different origin to a single chip with connection strategy $S_{h f}$. 


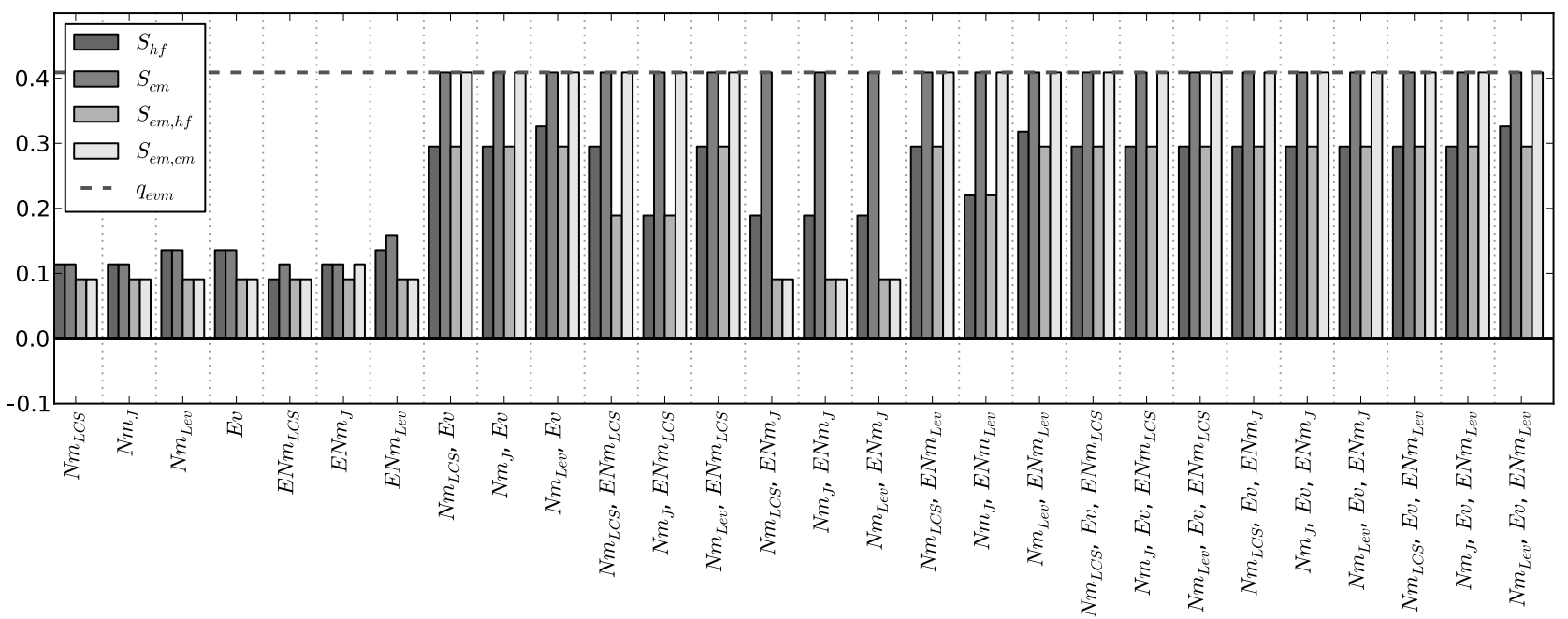

Figure 4. The $q$-value of the AES-design for different heuristics and connection strategies

\section{LIMITATIONS AND FUTURE WORK}

One goal of this work is to understand the limitations of the light-weight techniques. In this section we discuss the limitation we have found during the evaluation and possible ideas for future improvements. Our evaluation showed that a good naming convention helps the approach to achieve very good results, like in the case of the Wishbone-interface for which a very strict naming convention is applied and a perfect result is achieved. Also in case of the OpenSPARC core, where a naming convention is used to allow efficient use of the Emacs Verilog-Mode, the results are very good. However, the presented approach suffers from poor naming conventions.

Further, the approach suffers from its inability to create glue logic. Especially, the SD Mass Storage Controller uses multiplexer schemes to connect outputs of several sub-modules to one single input. Similarly, several designs use the concatenation of several signals as inputs to a sub-module.

We propose two advanced approaches for future work: First, a functional analysis that matches trigger conditions of one module with output sequences of other components. Second, validating the result of the automatically connected design with respect to the specification. Finally, as seen for the tiny-AES design, further work is required with respect to preventing incorrect connections.

\section{CONCLUSION}

In this paper we have presented an approach for automatically creating the connections between different logic blocks. The presented approach does not apply computationally expensive functional analysis. Instead our approach uses several heuristics which either mark a connection as invalid or assign a score to the connections based on a string metric to decide whether they are likely to be correct.

We compared our approach against Emacs Verilog-Mode with respect to eight different designs of various sizes. Our approach achieved better results for seven of those designs and an equally good result for the eighth design. Especially, our approach was able to achieve better results for the design which was optimized for being used with Emacs Verilog-Mode. Our approach generated a perfect connection in case of the Wishbone-interface a standardized interface to connect different blocks.

Further, the evaluation showed the limitations of the light-weight techniques and thus the cases for which more advanced approaches have to be considered.

\section{REFERENCES}

[1] "MKTREE," accesss date: 12.09.2013. [Online]. Available: http: //www.angelfire.com/biz/mktree/

[2] W. Snyder, "Verilog-mode: Reducing the veri-tedium," in Synopsys Users Group Conference, San Jose, 2001.

[3] "IEEE Standard for System Verilog- Unified Hardware Design, Specification, and Verification Language," IEEE Std 1800-2005, pp. 1-648, 2005.

[4] R. Zimmermann and R. Whitby, "Emacs VHDL mode 3.34," 2012 accesss date: 05.03.2014. [Online]. Available: http://www.iis.ee.ethz.ch/ $\sim$ zimmi/emacs/vhdl-mode.html

[5] C. Neely, G. Brebner, and W. Shang, "ShapeUp: A High-Level Design Approach to Simplify Module Interconnection on FPGAs," in IEEE Annual International Symposium on Field-Programmable Custom Computing Machines, 2010, pp. 141-148.

[6] "IEEE Standard for IP-XACT, Standard Structure for Packaging, Integrating, and Reusing IP within Tools Flows," IEEE Std 1685-2009, pp. C1-360, 2010.

[7] K. Avnit, V. D'Silva, A. Sowmya, S. Ramesh, and S. Parameswaran, "A formal approach to the protocol converter problem," in Design, Automation and Test in Europe, 2008, pp. 294-299.

[8] S. Williams, "Icarus Verilog," access date: 05.03.2014. [Online]. Available: http://iverilog.icarus.com

[9] W. E. Winkler, "String comparator metrics and enhanced decision rules in the fellegi-sunter model of record linkage." in Survey Research Methods Section, American Statistical Association, 1990, pp. 354-359.

[10] V. I. Levenshtein, "Binary codes capable of correcting deletions, insertions and reversals," in Soviet physics doklady, vol. 10, 1966, pp. 707-710.

[11] H. Hsing, "sha3," 2013, access date: 21.05.2013. [Online]. Available: http://opencores.org/project, sha3

[12] W. D. Peterson, Wishbone B4, OpenCores Std., 2010, access date: 05.03.2014. [Online]. Available: http://cdn.opencores.org/downloads/ wbspec_b4.pdf

[13] A. Edvardsson, “sdcard_mass_storage_controller," 2010, access date 21.05.2013. [Online]. Avvailable: http://opencores.org/project,sdcard_ mass_storage_controller

[14] D. Annayya, "uart2spi," 2013, access date: 21.05.2013. [Online] Available: http://opencores.org/project, uart2spi

[15] H. Hsing, "tiny_aes," 2013, access date: 21.05.2013. [Online]. Available: http://opencores.org/project,tiny_aes

[16] L. Wei, "mips789," 2009, accesss date: 21.05.2013. [Online]. Available: http://opencores.org/project,mips 789

[17] A. Edvardson, M. Erlandson, J. Baxter, R. D'Addio, J. Bennet, S. Fielding, M. Unneback, O. Kindgren, R. Herveille, Y. Vernier, J. Bonn, S. Kristiansson, S. Kim, P. Skrzypek, N. Anastasiadis, T. Markovic, A. Kamath, P. Gavin, and G. Scrivano, "OpenRISC 1200," 2012, accesss date: 17.06.2013. [Online]. Available: http: //opencores.org/or1k/Main_Page

[18] Sun Microsystems, "OpenSPARC T1," 2006, accesss date 03.07.2013. [Online]. Available: http://www.oracle.com/technetwork/ systems/opensparc/opensparc-t1-page-1444609.html 